\title{
Peertechz
}

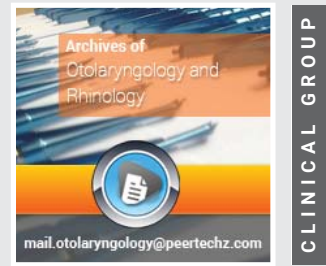

Case Report

\section{Treatment of sinusitis}

associate with filling material

of the maxillary sinus by

\section{endonasal endoscopic sinus}

surgery simultaneous sinus-

\section{lifting and dental implantation}

\section{Gagik Hakobyan ${ }^{1 *}$, Grigor Khachatryan², Levon Khachatryan³, Lazar Yessayan ${ }^{4}$ and Davit Mathevosyan ${ }^{5}$}

'Department of Oral and Maxillofacial Surgery, Yerevan State Medical University after M. Heratsi, Armenia

${ }^{2}$ Professor, Department of Oral and Maxillofacial Surgery, Yerevan State Medical University after M. Heratsi, Armenia

${ }^{3}$ Department of Maxillofacial and Plastic Surgery, Modern Implant Medicine, Armenia

${ }^{4}$ Professor, Head of Department of Therapeutic Dentistry, Yerevan State Medical University after M.

Heratsi, Armenia

${ }^{5}$ Assistant Professor of Department of Oral and Maxillofacial Surgery, Yerevan State Medical University after M. Heratsi, Armenia
Received: 25 September, 2020

Accepted: 21 November, 2020

Published: 23 November, 2020

*Corresponding author: Gagik Hakobyan, Department of Oral and Maxillofacial Surgery, Yerevan State Medical University after M. Heratsi, 0028 Kievyan str. 10 ap. 65 Yerevan, Armenia, Tel: (+37410)271146; E-mail:prom_hg@yahoo.com

Keywords: Sinus pathology; Endoscopic endonasal sinus surgery; Sinus lifting; Dental implantation https://www.peertechz.com

\section{Check for updates}

\section{Abstract \\ Objectives: Optimization of the sinus-lifting in patients with filling material in maxillary sinus cavity by a one-stage endonasal endoscopic elimination of the sinus pathology and carrying out a sinus-lifting.}

Materials and methods: A total of 14 patients (8 males and 6 females, the age was 31 to 64 years, from 2016 to 2020) with ridge defects in age group were selected for the study. All patients had a partially or totally edentulous atrophied posterior maxilla. All patients underwent a thorough clinical examination according to a generally accepted scheme.

For sinus surgery, used endoscope is $4.0 \mathrm{~mm}$ diameter rigid endoscope (Karl Storz). The fungal material, and hypertrophic mucosa within the maxillary sinus were removed and sent for pathological analysis. Sinus lifting procedures were performed using a lateral window approach.

A total of 23 sinus lifting procedures were performed, using a mixture of bovine bone, autogenous bone and PRP. According to our surgical procedure we performed in our patients 1 a 2 stage sinus lifting, 46 implants were inserted.Dental prosthetic rehabilitation was undertaken 5 months after implants insertion and submerged healing. Implant success was assessed clinically and radiographically. The height of the graft and bone density was measured 6th and 9th month after surgery using serial $\mathrm{CT}$. The following parameters were assessed: failure of the augmentation procedure, implant failure, and vertical bone height.

Results: Any intraoperative and postoperative complications, such asbleeding, membrane perforation, swelling, ecchymosis, pain, nasal bleeding, and infection, were recorded clinically and radiographically. Of the 46 implants placed in these 12 patients, 2 failed to osseointegrate. The CT examination showed the presence of dense 
bone around and above the implants. The implants appeared to be well integrated with no peri-implant bone loss. At 5 years follow up, excellent integration of grafted tissue, steady levels of bone around the implants and healthy peri-implant tissues were reported. Implants placed in the reconstructed areas were demonstrated to integrate normally, postoperative occlusal function and esthetics have been favorable.

Conclusion: The method of simultaneous endonasal sanitation of the maxillary sinus, sinus-lifting, dental implantation, allows to reduce the probability of perforation of the membrane, significantly shortening the rehabilitation period of patients with insufficient bone tissue in the maxillary sinus. These methods led to simpler, more comfortable, lower risks of morbidity, more predictable compared to more invasive maxillary sinus augmentation.

\section{Introduction}

At present, dental implants are the best solution for the rehabilitation of patients with various forms of toothless [1]. However, atrophy of the edentulous ridges make difficult for implant placement difficult.

Numerous procedures and materials are used to repair bone defects. The bone graft procedures used in oral implantology include autograft reconstruction, GBR, maxillary sinus floor elevation, and alveolar distraction osteogenesis [2-8]. The decision to choose any option depends on clinical factors and ultimately on the skill of the clinician.

The sinus lift procedure is one of the primary surgical options allowing placement of dental implants in the posterior maxilla. The traditional technique consists in a modified Caldwell-Luc approach, where access to maxillary sinus is obtained by drilling abone window in lateral sinus wall; then, Schneiderian membrane is carefully detached and elevated from sinus floor in order to insert grafting materials, including autogenous bone, allografts, xenografts, or alloplasts. Implants can be inserted simultaneously, or in a second stage if residual bone is not sufficient to obtain an adequate primary stability [9-13].

If the residual bone is $6-7 \mathrm{~mm}$, use the closed sinus lift method with access from the chewing surface of edentulous ridges using osteomas [14].

Sinus floor augmentation with autogenus bone grafts or with biomaterials has since long been the predominant, welldocumented procedure in the literature $[15,16]$. However, the procedure may be complicated in patients with chronic maxillary sinusitis $[17,18]$. Sinus membrane pathology can potentially complicate the post procedural course of sinus lift. In clinical practice, chronic maxillary sinusitis is often observed due to the hit of filling material from the tooth canal into the sinus cavity [19]. To remove the filling material from the sinus cavity, the traditional Caldwell-Luc method was used. Modern tendencies of oral surgery are aimed at minimizing surgical trauma and reducing the time for rehabilitation of patients. In this connection, new technologies without perforation violating the vestibular sinus wall which allow to reduce the volume surgical intervention and shorten the time of treatment. Revision sinus surgery for inflammatory diseases of maxillary sinus has been revolutionized by endoscopic techniques used in maxillary sinus surgery $[20,21]$.

Recent technological advances in the field of endoscopy have resulted in substantial improvements in endoscopecontrolled surgery of paranasal sinuses. Endoscopically technique involves endonasal approach by endoscop and is a minimally invasive procedure[22-26].

The most important factor in sinus lift surgery is atraumatic detachment of the periosteum of the maxillary sinus membrane from the bony antrum-floor to the preparation of a mucoperiosteal flap to provide a reliable osseointegration of and bone regeneration around the grafting material, which can only take place with a fully intact periosteum

Treating maxillary sinus pathology by endoscopic approaches, prior to implant insertion and/or sinus augmentation, is crucial for a better outcome of the dental procedure. In patients with pathologies sinus due to hit of filling material from the tooth canal into the sinus cavity needing a sinus lift procedures, optimal solution using endoscopic technology $[26,27]$.

\section{Objectives}

Optimization of the sinus-lifting in patients with filling material in maxillary sinus cavity by a one-stage endonasal endoscopic elimination of the sinus pathology and carrying out a sinus-lifting.

\section{Materials and methods}

A total of 14 patients ( 8 males and 6 females, the age was 31 to 64 years, from 2016 to 2020) with ridge defects in age group were selected for the study. All patients had a partially or totally edentulous atrophied posterior maxilla. All patients underwent a thorough clinical examination according to a generally accepted scheme.

Preoperative planning includes a careful history and physical exam, in addition to preoperative radiologic investigation, which include computed tomography scan to to determine the existing osseos structure and to evaluate any pathology of the sinuses (Figure 1). Treatment initiates with the administration of a single preoperative dose of systemic antibiotic (Amoxicillin, clindamycin or levaquin) and Chlorhexidine 0.12 percent rinse.

A total of 18 sinus lifting procedures were performed, using a mixture of bovine bone, autogenous bone and PRP.

\section{Surgical technique}

For sinus surgery, used endoscope is $4.0 \mathrm{~mm}$ diameter rigid endoscope (Karl Storz). It provides different angles of vision in maxillary sinus from 0/30/45/70/90/ 2.7-mm/4.0-mm endoscopes (Karl Storz, Tuttlingen, Germany) and a monitor were used for inspection and treatment of the and a monitor were used for inspection and treatment of the maxillary sinus. 
The first procedure was always the endoscopic endonasal sinus surgery. This was performed in all the patients through an enlarged natural sinus ostium in the middle nasal meatus. All operations were carried out under general anesthesia. The ostium was enlarged to a size that allowed access to the sinus with appropriate instruments. The fungal material, and hypertrophic mucosa within the maxillary sinus were removed and sent for pathological analysis. Sinus lifting procedures were performed using a lateral window approach. Osteotomy was performed on the lateral surface of the sinus wall using a round drill. After the elevation of the sinus membrane, the dental implant sites were prepared using low-speed calibrated burrs, specific to the implant system used. The cavity between the sinus membrane and the sinus floor was filled in with a mixture of particulate bovine bone graft (BioOsss, Geistlich Pharma, Wolhusen, Switzerland), autologous bone, and plateletrich plasma (PRP), and the dental implants were inserted with a good primary stability. According to our surgical procedure we performed in our patients 1 a 2 stage sinus lifting, 46 implants were inserted.

The delayed establishment of dental implants was carried out in 4 patients after 5 months after operation. Immediate implant placement (one-stage sinus lifting protocol) was performed when a mean bone height of at least $4 \mathrm{~mm}$ was present on CT examination. For the one-stage protocol the implant site was prepared and the implant inserted in the residual subantral bone. The osteotomy window was covered with the PRP membrane before flap closure. The mucoperiostal flap was sutured using 3.0 silk suture. Hospitalization after surgery varied from 1 to 2 days. The sutures were removed $10 \mathrm{e} 14$ days postoperatively.

The height of the graft and bone density was measured 6th and 9th month after surgery using serial CT scan (Figure 2).

Dental prosthetic rehabilitation was undertaken 5 months after implants insertion and submerged healing. Implant success was assessed clinically and radiographically.

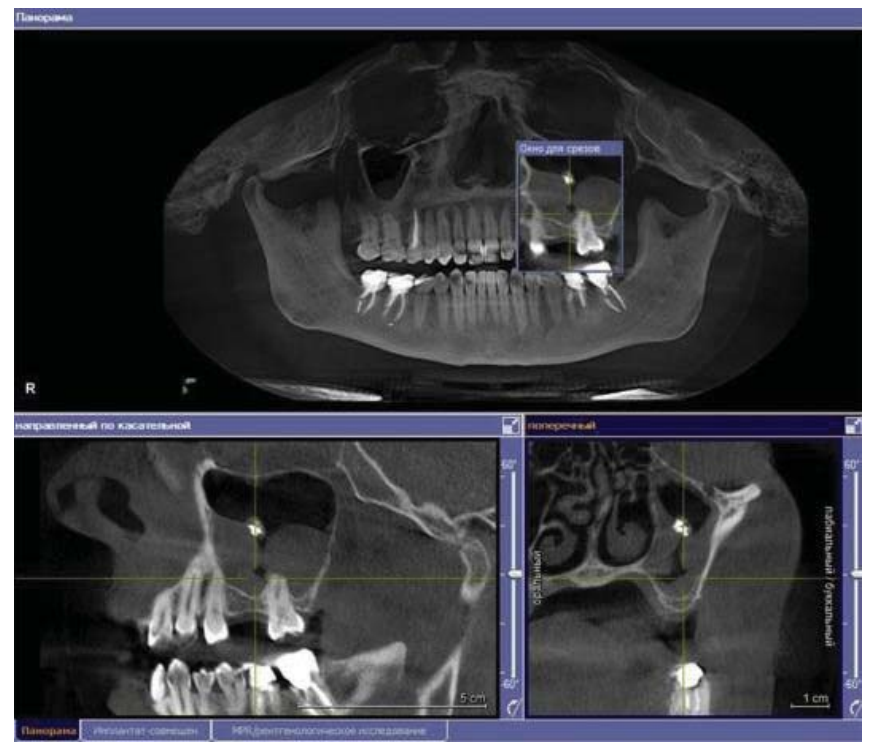

Figure 1: Preoperative CT scan of a patient with polyposive sinusitis, showing foreign bodies in the maxillary sinus (filling material).

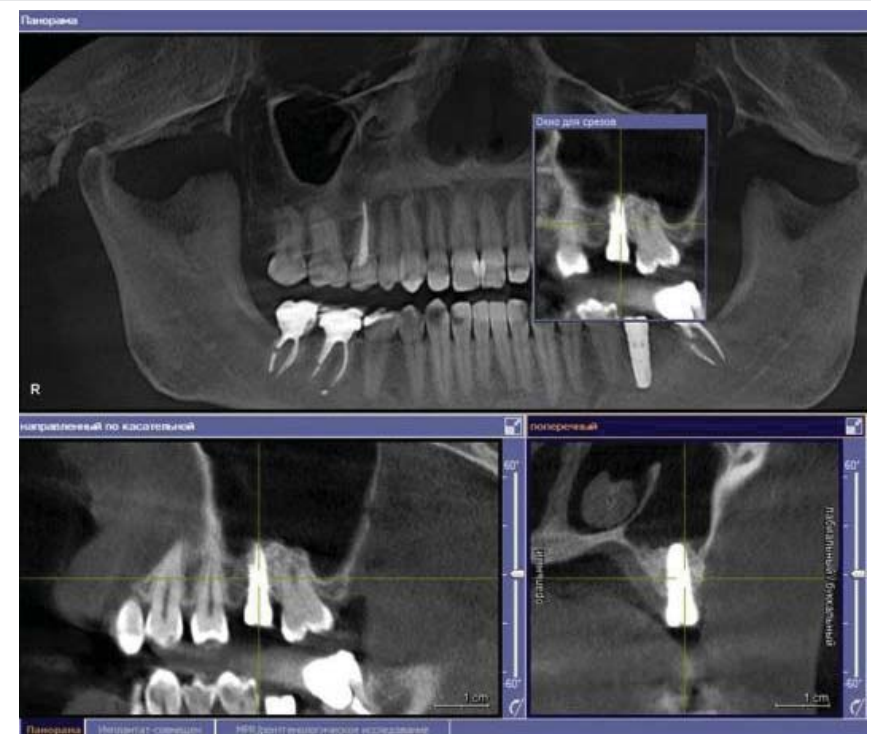

Figure 2: Postoperative CT scan of a patient with foreign body of the maxillary sinus (filling material) with polyposive sinusitis following simultaneous endonasal sanitation of the maxillary sinus, sinus-lifting, and dental implantation.

\section{Results}

Any intraoperative and postoperative complications, such asbleeding, membrane perforation, swelling, ecchymosis, pain, nasal bleeding, and infection, were recorded clinically and radiographically. The following parameters were assessed: failure of the augmentation procedure, implant failure, and vertical bone height.

Of the 46 implants placed in these 12 patients, 2 failed to osseointegrate. The CT examination showed the presence of dense bone around and above the implants. The implants appeared to be well integrated with no peri-implant bone loss. At 5 years follow up, excellent integration of grafted tissue, steady levels of bone around the implants and healthy periimplant tissues were reported. Satisfactory facial symmetry, chewing and speech functions of the patients were restored. Implants placed in the reconstructed areas were demonstrated to integrate normally, postoperative occlusal function and esthetics have been favorable.

The method of simultaneous endonasal sanitation of the maxillary sinus and dental implantation, allows to reduce the probability of perforation of the membrane, significantly shortening the rehabilitation time of patients with insufficient bone tissue in the area of the maxillary sinus.

As a result of the introduction of these innovative technologies, surgical technologies for managing patients with sinus pathology have been optimized, using minimally invasive endoscopic technique, simultaneous endonasal sanation of the maxillary sinus with endoscopic assisted sinus lifting before dental implantation.

\section{Conclusion}

The method of simultaneous endonasal sanitation of the maxillary sinus, sinus-lifting, dental implantation, allows 
to reduce the probability of perforation of the membrane, significantly shortening the rehabilitation period of patients with insufficient bone tissue in the maxillary sinus. These methods led to simpler, more comfortable, lower risks of morbidity, more predictable compared to more invasive maxillary sinus augmentation.

\section{Consent statement}

Written informed consent was obtained from the patient for publication of this case report and accompanying images.

\section{Ethical approval}

The study was reviewed and approved by the Ethics Committee of the of the Yerevan State Medical University after M. Heratsi (protocol N16, 5.10.17) and in accordance with those of the World Medical Association and the Helsinki Declaration. Informed consent Patients were informed verbally and in writing about the study and gave written informed consent.

\section{References}

1. Branemark PL, Hansson BO, Adell R, Breine U, Breine U, et al. (1977) Osseointegrated implants in the treatment of edentulous jaw. Experience from a 10-year period. Scand J Plast Reconstr Surg 2: 1-13. Link: https://bit.ly/3pNtYbw

2. Chiapasco M, Casentini $P$, Zaniboni M (2009) Bone Augmentation Procedures in Implant Dentistry. Int J Oral Maxillofac Implants 24: 237-259. Link: https://bit.ly/3kXxOLS

3. Harshakumar K, Varghese NM, Ravichandran R, Lylajam S (2014) Alveolar Ridge Augmentation using Autogenous Block Bone Grafts Harvested from Mandibular Ramus to Facilitate Implant Placement: A Case Report. International Journal of Scientific Study 2: 46-50. Link: https://bit.ly/36QQ5Fs

4. Lozano RA, Dominguez-Mompell JL, Infante-Cossio P, Lara-Chao J, LopezPizarro V (2015) Calvarial Bone Grafting for Three-Dimensi Reconstruction of Severe Maxillary Defects. Int J Oral Maxillofac Implants 30: 881-890. Link: https://bit.ly/38XHyDo

5. Levin B (2013) Alveolar Ridge Augmentation: Combining Bioresorbable Scaolds with Osteoinductive Bone Grafts in Atrophic Sites. A Follow-Up to an Evolving Technique. Compend Contin Educ Dent 34: 178-187. Link: https://bit.ly/36TpvLP

6. Contar M, Sarot JR, Bordini J, Galvão GH, Nicolau GV, et al. (2009) Maxillary Ridge Augmentation With Fresh-Frozen Bone Allografts. J Oral Maxillofac Surg 67: 1280-1285. Link: https://bit.ly/35UKb7b

7. Rachmiel A, Shilo D, Aizenbud D, Emodi O (2017) Vertical Alveolar Distraction Osteogenesis of the Atrophic Posterior Mandible Before Dental Implant Insertion. J Oral Maxil fac Surg 75: 1164-1175. Link: https://bit.ly/3pRYu4m

8. Aloja DE, Ricci M, Caso G, Santi E, Paolo T, et al. (2013) The use of bone block allografts in sinus augmentation,followed by delayed implant placement: Case Series. Contemp Clin Dent 4: 13-19. Link: https://bit.ly/36Sk9Re

9. Boyne PJ, James RA (1980) Grafting of the maxillary sinus floor with autogenous marrow and bone. J Oral Surg 38: 613-616. Link: https://bit.ly/2UJdNOe
10. Manev G, Popova C (2013) Sinus flor augmentation with bone blok grafting and simulatneous implant placment .Journal of IMAB 19: 348-354. Link: https://bit.ly/2IXt37H

11. Misch CE (1999) The maxillary sinus lift and sinus graft surgery. In: Contemporary implant Dentistry. St Louis: Mosby 469-495.

12. Peleg M, Mazor Z, Chaushu G, Garg AK (1988) Sinus floor augmentation with simultaneous implant placement in the severely atrophic maxilla. J Periodontol 69: 1397-1403. Link: https://bit.ly/3fib87E

13. Block MS, Kent JN (1997) Sinus augmentation for dental implants, the use of autogenous bone. J Oral MaxillofacSurg 55: 1281-1286. Link: https://bit.ly/3nHC7MX

14. Tatum H (1986) Maxillary and sinus implant reconstruction. Dent Clin North Am 30: 207-229. Link: https://bit.ly/394o4gb

15. Chanavaz M (2000) Sinus graft procedures and implant dentistry: a revien of 21 years of surgical experience. Implant Dent 3: 197-203. Link: https://bit.ly/35MyBL8

16. Winter AA, Pollack AS, Odrich RB (2002) Placement of implants in the severly atrophic posterior maxilla using localized manegement of the sinus floor:Apreliminary stady. Int J Oral Maxillofac Implants 17: 687-695. Link: https://bit.ly/2KtaE3j

17. Schwartz-Arad D, Herzberg R, Dolev E (2004) The prevalence of surgical complications of the sinus graft procedure and their impact on implant survival. J Periodontol 75: 511-516. Link: https://bit.ly/3nCWDya

18. Bhattacharya N (1999) Bilateral chronic maxillary sinusitis after the sinus lift procedure. Am J Otolaryngol 20: 133-135. Link: https://bit.ly/2Ku4ZKk

19. Mehra P, Murad H (2004) Maxillary sinus disease of odontogenic origin. Otolaryngol Clin North Am 32: 2347-2364. Link: https://bit.ly/334CkSG

20. Kennedy DW (1985) Functional Endoscopy sinus surgery technique. Arch Otolaryngol 111: 643-649. Link: https://bit.ly/3fhLJet

21. Jiang RS, Hsu CY (2002) Revision functional endoscopic sinus surgery. Ann Otol Rhinol Laryngol 111: 155-159. Link: https://bit.ly/3ISKvbM

22. Tajudeen BA, Kenned DW (2017) Thirty years of endoscopic sinus surgery What have we learned?. World J Otorhinolaryngol Head Neck Surg 3: 115-121. Link: https://bit.ly/2UJ1cuB

23. Bunzen DL, Campos A, Leão FS, Morais A, Sperandio F, et al. (2006) Efficacy of functional endoscopic sinus surgery for symptoms in chronic rhinosinusitis with or without polyposis. Braz J Otorhinolaryngol 72: 242-246. Link: https://bit.ly/36XxLui

24. Kennedy DW (2006) Technical innovations and the evolution of endoscopic sinus surgery. Ann Otol Rhinol Laryngol 196: 3-12. Link: https://bit.ly/2UJ1q4V

25. Busaba NY, Kieff D (2002) Endoscopic sinus surgery for inflammatory maxillary sinus disease. Laryngoscope 112: 1378-1383. Link: https://bit.ly/2IUEAVa

26. Costa F, Emanuelli E, Robiony M, Zerman N, Polini F, et al. (2007) Endoscopic surgical treatment of chronic maxillary sinusitis of dental origin. $\mathrm{J}$ Oral Maxillofac Surg 65: 223-228. Link: https://bit.ly/3ISKGns

27. Khachatryan L, Khachatryan G, Hakobyan G, Khachatryan A (2019) Simultaneous endoscopic endonasal sinus surgery and sinus augmentation with immediate implant placement: A retrospective clinical study of 23 patients. J Craniomaxillofac Surg 47: 1233-1241. Link: https://bit.ly/35QdiZe

Copyright: @ 2020 Hakobyan G, et al. This is an open-access article distributed under the terms of the Creative Commons Attribution License, which permits unrestricted use, distribution, and reproduction in any medium, provided the original author and source are credited.

Citation: Hakobyan G, Khachatryan G, Khachatryan L, Yessayan L, Mathevosyan D (2020) Treatment of sinusitis associate with filling material of the maxillary sinus by endonasal endoscopic sinus surgery simultaneous sinus-lifting and dental implantation. Arch Otolaryngol Rhinol 6(4): 114-117. 